\title{
MODULATIONAL STABILITY OF KORTEWEG-DE VRIES AND BOUSSINESQ WAVETRAINS
}

\author{
BHIMSEN K. SHIVAMOGGI \\ Physical Research Laboratory \\ Ahmedabad - 380009 , INDIA \\ and
}

\section{LOKENATH DEBNATH}

Department of Mathematics University of Central Florida Orlando, Florida 32816, U.S.A.

(Received in October 1981 and in revised form November 12, 1983)

ABSTRACT. The modulational stability of both the Korteweg-de Vries (KdV) and the Boussinesq wavetrains is investigated using Whitham's variational method. It is shown that both $\mathrm{KdV}$ and Boussinesq wavetrains are modulationally stable. This result seems to confirm why it is possible to transform the $\mathrm{KdV}$ equation into a nonlinear Schrödinger equation with a repulsive potential. A brief discussion of Whitham's variational method is included to make the paper self-contained to some extent.

KEY WORDS AND PHRASES: Nonlinear Waves, Whitham's variational principle, KdV and Boussinesq wavetrains, modulational stability, nonlinear Schrödinger equation. 1980 MATHEMATICS SUBJECT CLASSIFICATION CODES : 76E30, 76B25,76B15.

\section{INTRODUCTION :}

In recent years, considerable attention has been given to nonlinear stability analysis of periodic wavetrains in fluids and plasmas. The complete investigation of the instability of periodic wavetrains on the surface of water was carried out independently by Lighthill [1], Whitham [2] and Benjamin [3]. Their theoretical and experimental investigation is now regarded as conclusive evidence of the instability of Stokes waves in deep water. This instability phenomenon led to the question of the evolution of weakly nonlinear wavetrains.

For weakly nonlinear waves, the evolution of a wavetrain is described by Whitham's conservation equations which consist of the conservation of wavenumber, the conservation of wave action, and the dispersion relation in the form

$$
\begin{gathered}
\frac{\partial k}{\partial t}+\frac{\partial \omega}{\partial x}=0 \\
\frac{\partial a^{2}}{\partial t}+\frac{\partial}{\partial x}\left(\frac{\partial \omega}{\partial k} a^{2}\right)=0
\end{gathered}
$$




$$
\omega=\sqrt{g k}\left(1+\frac{1}{2} k^{2} a^{2}\right)
$$

where $k$ is the wavenumber, $\omega$ is the frequency and $a$ is the amplitude of the wavetrain. These equations are the leading order equations derivable from the Whitham averaged variational principle [4].

In order to investigate the evolution of Stokes waves in deep water, Chu and Mei [5-6] derived the modulation equations of Whitham's type for slowly varying waves as

$$
\begin{gathered}
\frac{\partial a^{2}}{\partial t}+\frac{\partial}{\partial x}\left(-\frac{1}{2} w a^{2}\right)=0 \\
\frac{\partial W}{\partial t}+\frac{\partial}{\partial x}\left(-\frac{1}{4} w^{2}+\frac{1}{4} a^{2}+\frac{a x x}{16 a}\right)=0,
\end{gathered}
$$

Based upon these equations, they also conducted a numerical study of the nonlinear evolution of wave envelope in deep water. It was found that the wave envelope tends to disintegrate to multiple groups of waves each of which approaches a stable permanent envelope representing dynamical equilibrium.

It is interesting to point out that equations $(1.4)$ - (1.5) can be combined to obtain the nonlinear Schrodinger equation. To prove this claim, we introduce a small phase variation $\phi$ defined by $W=-2 \phi_{x}$ so that (1.5) becomes

$$
-2 \phi_{x t}+\frac{\partial}{\partial x}\left(-\phi_{x}^{2}+\frac{1}{4} a^{2}+\frac{a x x}{16 a}\right)=0
$$

We now integrate (1.6) with respect to $x$ and set the constant of integration to be zero to transform (1.6) in the form

$$
\phi_{t}+\frac{1}{2} \phi_{x}^{2}-\frac{1}{8} a^{2}-a_{x x} / 32 a=0
$$

Substituting $\psi=\mathrm{a} \exp (4 i \phi)$ into (1.4) and (1.7), we find the nonlinear Schrödinger equation

$$
i \psi_{t}+\frac{1}{8} \psi_{x x}+\frac{1}{2} \psi|\psi|^{2}=0
$$

For the small amplitude equations of Whitham's theory of slowly varying wavetrains including the effects of dissipation, it was shown by Davey [7] that the modulation of the wave is described by a more general nonlinear Schrödinger equation,

$$
i \psi_{t}+\frac{1}{2} \gamma \psi_{x x}-\alpha|\psi|^{2} \psi-i \Omega_{o i} \psi=0
$$

where $\alpha$ and $\gamma$ are complex constants and the last term on the left hand side represents dissipation.

Davey discussed a number of new and interesting points concerning the propagation of weak nonlinear waves. It was shown that equation (1.9) reduces to the KdV equation for purely dispersive long waves $(k \rightarrow 0)$. amd to the Burgers equation for long dissipative waves.

Taniuti and Wei [8] have presented a class of nonlinear partial differential equations which admit a reduction to tractable nonlinear equations such as the $\mathrm{KdV}$ and the Burgers equations. Their method of reduction is based on a singular perturbation expansion. They have shown that it is possible to transform the KdV equation into a nonlinear Schrödinger equation with a repulsive potential which is known not to lead to a modulational instability. 
Motivated by the above discussion, we find it interesting to examine the problems of modulational stability further. So the purpose of the present paper is to demonstrate the modulational stability of both $\mathrm{KdV}$ and Boussinesq wavetrains. We shall use Whitham's variational method.

\section{WHITHAM'S VARIATIONAL METHOD}

In his pioneering work, Whitham [4] developed a general variational method to describe slow variations of amplitude, frequency, and wavenumber of nonuniform nonlinear wavetrain using a Lagrangian. In fact, he takes an average over the local oscillations in the medium, and prescribes an averaged variational principle with an appropriate Langrangian.

In cases where the governing equations admit uniform periodic wavetrains as solutions, Whitham [4] pointed out that the system can be derived from a Hamilton variational principle of the form,

$$
\delta \iint L\left(u_{t}, u_{x}, u\right) d x d t=0
$$

where $L$ is the Lagrangian of the system and the dependent variable $u \equiv u(x, t)$.

It follows from (2.1) that the Euler - Lagrange equation is

$$
\frac{\partial}{\partial t}\left(\frac{\partial L}{\partial u_{t}}\right)+\frac{\partial}{\partial x}\left(\frac{\partial L}{\partial u_{x}}\right)-\frac{\partial L}{\partial u}=0
$$

This is a second order partial differential equation for $u(x, t)$. If the equation has a periodic solution of the form

$$
\mathrm{u}=\mathrm{U}(\Theta), \quad \Theta=\mathrm{kx}-\omega \mathrm{t}
$$

it turns out that three parameters $\omega, k, a$ are connected by a nonlinear dispersion relation

$$
D(\omega, k, a)=0
$$

In linear problems with the wavetrain solution $u=U(\Theta)=a e^{i \Theta}$, the dispersion relation becomes independent of a.

For slowly varying dispersive wavetrains, the solution maintains the elementary form (2.4ab) but $\omega, k$, and a are no longer constants so that $\Theta$ is not a linear function of $x$ and $t$. The local wavenumber and frequency are defined by

$$
k=\theta_{x}, \omega=-\theta_{t} \text {. }
$$

The parameters $\omega, k$ and $a$ are slowly varying functions of $x$ and $t$. Thus (2.6ab) leads to a compatibility condition

$$
\frac{\partial k}{\partial t}+\frac{\partial \omega}{\partial x}=0
$$

This is indeed an equation for conservation of waves.

If the period of the function $u=U(\theta)$ is normalized to $2 \pi$, the averaged Lagrangian is defined by

$$
\mathcal{L}(\omega, k, a)=\frac{1}{2 \pi} \int_{0}^{2 \pi} L d \theta .
$$

Then the Whitham averaged variational principle is given by

$$
\delta \iint \mathcal{L}(\omega, \mathrm{k}, \mathrm{a}) \mathrm{dt} d \mathrm{x}=0
$$


This equation is then used to derive equations governing $\omega, k$ and $a$.

The Euler equations resulting from the independent variations $\delta a$ and $\delta \Theta$ are

$$
\begin{array}{cc}
\delta \mathrm{a}: & \frac{\partial \mathcal{L}}{\partial \mathrm{a}}=0 \\
\boldsymbol{\delta} \ni: & \frac{\partial}{\partial t}\left(\frac{\partial \mathcal{L}}{\partial \omega}\right)-\frac{\partial}{\partial \mathrm{x}}\left(\frac{\partial \mathcal{L}}{\partial \mathrm{k}}\right)=0
\end{array}
$$

Equation (2.10) turns out to be the dispersion relation.

3. MODULATIONAL STABILITY OF KdV WAVETRAINS

We consider the $\mathrm{KdV}$ equation in the form

$$
\phi_{t}+\alpha \phi \phi_{x}+\beta \phi_{x x x}=0
$$

where $\phi=\phi(x, t)$ is the dependent variable.

This has the variational characterization

$$
\delta \iint \operatorname{Ldt} \mathrm{dx}=0
$$

where the Lagrangian $\mathrm{L}$ is given by

$$
\mathrm{L} \equiv \frac{1}{2} \psi_{t} \psi_{x}+\frac{1}{6} \alpha \psi_{x}^{3}-\frac{1}{2} \beta \psi_{x x}^{2}, \psi_{x}=\phi,
$$

For a weakly nonlinear slowly - varying oscillatory waretrain, we consider a solution of the form

$$
\psi \equiv \Psi(\theta)=a(x, t) e^{i \Theta(x, t)}
$$

where $\theta_{x}=k(x, t)$ and $\theta_{t}=-\omega(x, t)$ are slowly - varying functions of $x$ and $t$, and still have the significance of a local wavenumber and local frequency.

Using (3.4), the expression for $L$ assumes the form

$$
\mathrm{L}=\frac{1}{2} \mathrm{k}\left(\frac{1}{3} \alpha \mathrm{k}^{2} \Psi_{\Theta}-\omega\right) \Psi_{\Theta}^{2}-\frac{1}{2} \beta \mathrm{k}^{4} \Psi_{\Theta \Theta}^{2}
$$

We assume

$$
\Psi(\theta)=\frac{b \Theta}{k}+a \cos \theta+a_{2} \operatorname{Cos} 2 \theta+\ldots
$$

where each term is of the higher order than the preceeding one.

In order to derive equations governing $\omega, k, a_{1}, a_{2}$, we take the average of the Lagrangian $L$ over the local oscillations, treating $\omega, k, a_{1}, a_{2}$ as constants. (This is in the spirit of Krylov - Bogliubov method. Bogliubov and Mitropolski [8] assume the average over the fast time scale in order to obtain equations governing the slowly - varying quantities). It turns out

where $L$ is given by (3.5).

$$
\bar{L}=\frac{1}{2} \pi \int_{0}^{2 \pi} L d \Theta
$$

In view of $(3.6)$, result (3.7) gives $_{2}$

$$
\overline{\mathrm{L}}=\frac{1}{2} \mathrm{k}\left(\frac{1}{3} \alpha b \mathrm{~b}-\omega\right) \frac{\mathrm{b}^{2}}{\mathrm{k}^{2}}+\frac{1}{2} \mathrm{k}(\alpha \mathrm{bk}-\omega) \frac{\mathrm{a}_{1}}{2}+\mathrm{k}\left(\alpha b \mathrm{~b}-\omega-4 \beta \mathrm{k}^{3}\right) \mathrm{a}_{2}-\frac{1}{2} \mathrm{a}_{1}{ }^{2} \mathrm{a}_{2}+\ldots
$$

We next apply the Whitham averaged variational principle

$$
\delta \overline{\mathcal{L}}=8 \iint \overline{\mathrm{L}}\left(\omega, \mathrm{k}, \mathrm{a}_{1}, \mathrm{a}_{2}, \ldots\right) \mathrm{dt} \mathrm{dx}=0
$$

This gives the following results corresponding to variations $\delta a_{1}, \delta a_{2}, \ldots$ and $\delta \ominus$ : 


$$
\begin{gathered}
\delta a_{1}: \quad \frac{\partial \mathcal{L}}{\partial a_{1}}=0 \\
\delta a_{2}: \quad \frac{\partial \mathcal{L}}{\partial a_{2}}=0 \\
\delta \Theta: \quad \frac{\partial}{\partial t}\left(\frac{\partial \mathcal{L}}{\partial \omega}\right)-\frac{\partial}{\partial x}\left(\frac{\partial \mathcal{L}}{\partial k}\right)=0
\end{gathered}
$$

It follows from equation (3.11) that

$$
a_{2}=a_{1}^{2} /\left[24 k\left(\alpha b k-\omega-4 \beta k^{3}\right)\right]
$$

Using (3.3), the expression for $\bar{L}$ in (3.8) takes the form $\bar{L}=\frac{b^{2}}{2 k}\left(\frac{1}{3} \alpha b k-\omega\right)+\frac{1}{4} k a_{1}{ }^{2}\left(\alpha b k-\omega-\beta k^{3}\right)-\left(\alpha k^{3}\right)^{2} a_{1}^{4} /\left[24 \times 24 k\left(a b k-\omega-4 \beta k^{3}\right)\right](3.14)$

This result can be used to obtain the explicit form of the dispersion relation (3.10) as

$$
\left(\alpha b k-\omega-\beta k^{3}\right)-\left[\frac{8}{24 \times 24 k^{2}\left(\alpha b k-\omega-4 \beta k^{3}\right)}\right] a_{1}{ }^{2}=0,
$$

It is interesting to note that the first part in (3.15) corresponds to the linearized problem for the KdV equation, and the amplituds - dependent part in (3.15) represents the nonlinear effects. It also follows from (3.15) that

$$
\omega \approx\left(\alpha b k-\beta k^{3}\right)+\frac{\alpha^{2} k}{216 \beta} a_{1}^{2} \text {. }
$$

If the disparsion relation for a nonlinear dispersive wave is of the form

$$
\omega=\omega_{0}(k)+\omega_{2}(k) a^{2}
$$

then, according to Whitham's theory, the waves in question are modulationally unstable if $\omega_{2}<0$.

For the present case dealing with the KdV wavetrains, we obtain from (3.16):

$$
\omega_{2}=\frac{\alpha^{2} k}{216 \beta}>0 \text {. }
$$

This confirms the fact that the $\mathrm{KdV}$ wavetrain's are modulationally stable. This seems to explain why it is possible to transform the $\mathrm{KdV}$ equation into a nonlinear Schrödinger equation with a repulsive potential [8] which is known not to lead to a modulational instability.

4. MODULATIONAL STABILITY OF BOUSSINESQ WAVETRAINS

We apply Whitham's variational method to the Boussinesq equation for investigation of modulational stability. We write the Boussinesq equation in the form

$$
\phi_{t t}-\phi_{x x}-\phi_{x x x x}-\left(\phi^{2}\right)_{x x}=0 \text {. }
$$

This has the following variational representation

$$
\delta \iint L d t d x=0
$$

where the Lagrangian $\mathrm{L}$ is

$$
\mathrm{L}=\frac{1}{2} \psi_{t \mathrm{t}} \psi_{\mathrm{xx}}-\frac{1}{2} \psi_{\mathrm{xx}}^{2}+\frac{1}{2} \psi_{\mathrm{xxx}}^{2}+\frac{1}{3} \psi_{\mathrm{xx}}^{3}, \quad \phi=\psi_{\mathrm{xx}},
$$

For a weakly-nonlinear slowly-varying wavetrain, we assume a solution of the form

$$
\psi=\Psi(\Theta)=a e^{i \Theta},
$$


where $\Theta_{x}=k(x, t),-\Theta_{t}=\omega(x, t)$, and $a=a(x, t)$ are slowly - varying function of $x$ and $t$.

In view of (4.4), the expression (4.3a) for $L$ has the form

$$
L=\frac{1}{2} k^{2}\left(\omega^{2}-k^{2}\right) \psi_{\Theta \Theta}^{2}+\frac{1}{3} k^{6} \Psi_{\Theta \Theta}^{3}+\frac{1}{2} k^{6} \Psi_{\Theta \Theta \Theta}^{2}
$$

We also assume the expansion of $\Psi(\theta)$ as

$$
\Psi(\Theta)=a_{1} \cos \theta+a_{2} \cos 2 \theta+\ldots \ldots
$$

where each term is of higher order than the preceeding one.

A procedure similar to that described by results (3.7) - (3.8) yields

$$
\bar{L}=\frac{1}{2 \pi} \int_{0}^{2 \pi} L d \Theta
$$

where

$$
\overline{\mathrm{L}}=\frac{1}{2} \mathrm{k}^{2}\left(\omega^{2}-\mathrm{k}^{2}\right)\left(\frac{1}{2} \mathrm{a}_{1}^{2}+8 \mathrm{a}_{2}^{2}\right)+\mathrm{k}^{6} \mathrm{a}_{1}{ }^{2} \mathrm{a}_{2}+\frac{1}{2} \mathrm{k}^{6}\left(\frac{1}{2} \mathrm{a}_{1}^{2}+32 \mathrm{a}_{2}^{2}\right)+\ldots \ldots
$$

As before, we apply the Whitham averaged variational principle

$$
\delta \iint \bar{L}\left(\omega, k, a_{1}, a_{2} \ldots\right) d t d x=0
$$

from which one can obtain the following results corresponding to variations $\delta a_{1}, \delta a_{2}$ $\ldots$ and $\delta \theta$ :

$$
\begin{gathered}
\delta \mathrm{a}_{1}: \quad \frac{\partial \mathcal{L}}{\partial \mathrm{a}_{1}}=0 \\
\delta \mathrm{a}_{2}: \quad \frac{\partial \mathcal{L}}{\partial \mathrm{a}_{2}}=0 \\
\cdot \cdot \cdot \cdot \cdot \cdot \cdot \cdot \cdot \cdot \frac{\partial}{\partial \mathrm{x}}\left(\frac{\partial \mathcal{L}}{\partial \mathrm{k}}\right)=0
\end{gathered}
$$

Equation (4.11) gives

$$
a_{2}=-\frac{1}{24} a_{1}^{2}
$$

so that (4.8) becomes

$$
\bar{L}=\left[\frac{1}{2} k^{2}\left(\omega^{2}-k^{2}\right)+\frac{k^{6}}{2}\right] \frac{a_{1}^{2}}{2}-\left[-\frac{k^{2}}{144}\left(\omega^{2}-k^{2}\right)+\frac{k^{6}}{24}-\frac{k^{6}}{36}\right] a_{1}^{4}
$$

This result is used to deduce the explicit form of the dispersion relation (4.10) as

$$
\left(\omega^{2}-k^{2}+k^{4}\right)-\frac{k^{4}}{6} a_{1}^{2}=0
$$

It is noted that the first term in (4.15) corresponds to the linearized dispersion relation, and the second term in (4.15) represents the nonlinear effects. It follows from (4.15) that

$$
\omega \approx\left(k^{2}-k^{4}\right)^{\frac{1}{2}}+\frac{k^{4}}{12\left(k^{2}-k^{4}\right)^{\frac{1}{2}}} a_{1}^{2}
$$

Comparing this result with (3.17), we conclude that

$$
\omega_{2}=\frac{k^{4}}{12\left(k^{2}-k^{4}\right)^{\frac{1}{2}}}>0
$$

This means that the Boussinesq wavetrains are also modulationally stable. 


\section{REFERENCES}

1. LIGHTHILL, M.J., Contributions to the theory of waves in nonlinear dispersive systems, J. Inst. Math. App1. 1 (1965) 269-306.

2. WHITHAM, G.B., Nonlinear Dispersion of water waves, J. F1uid Mech. 27 (1967) 399-412.

3. BENJAMIN, T.B., Instability of Periodic wavetrains in nonlinear dispersive systems, Proc. Roy. Soc. London 299A (1967) 59-75.

4. WHITHAM, G.B., Linear and Nonlinear Waves, Wiely- Interscience, New York, 1974

5. CHU, V.H., and MEI, C.C., On Slowly-varying Stokes waves, J. Fluid Mech. 41 (1970) 873-887

6. CHU, V.H., and MEI, C.C., The evolution of Stokes waves in deep water, J. Fluid Mech. 47 (1971) 337-351.

7. DAVEY, A., The propagation of a weak nonlinear wave, J. Fluid Mech. 53 (1972) 769-78

8. TANIUTI, T., and WEI, C.C., Reductive Perturbation Method in Nonlinear Wave Propagation I., J. Phys. Soc. Japan 24 (1968) 941-946.

9. BOGLIUBOV, M.N., and MITROPOLSKI, Y.A., Asymptotic Methods in the Theory of Nonlinear Oscillations, Hindustan Publishing Co., New Delhi (1961).

10. DEBNATH, L., Nonlinear Waves, Cambridge University Press, Cambridge (1983)

Present address of Bhimsen K. Shivamoggi

Institute of Mathematical Sciences

Madras - 600 113, India 


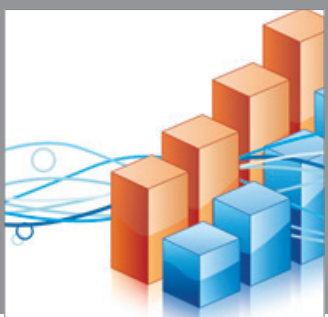

Advances in

Operations Research

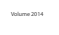

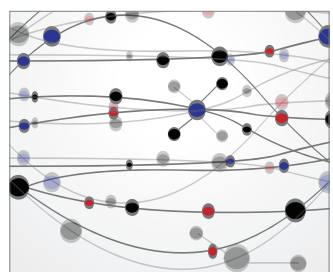

\section{The Scientific} World Journal
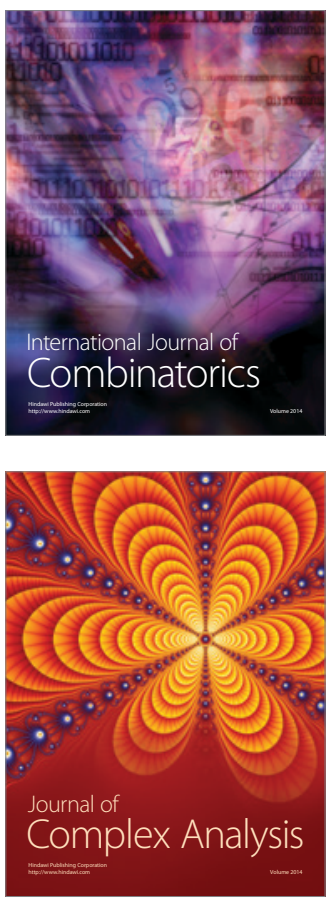

International Journal of

Mathematics and

Mathematical

Sciences
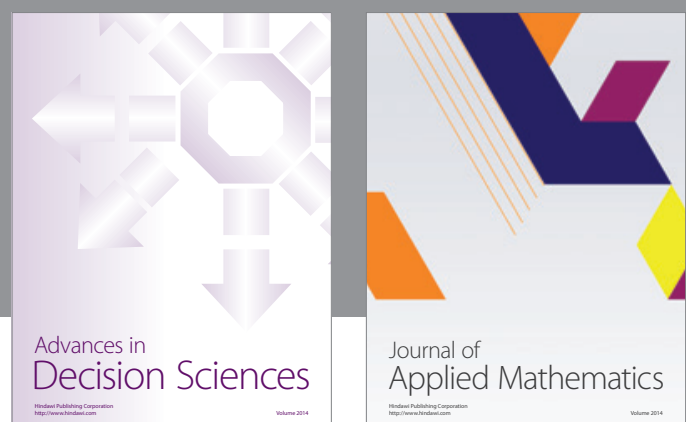

Journal of

Applied Mathematics
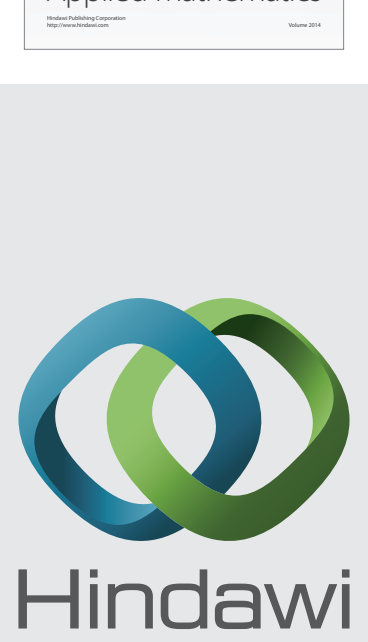

Submit your manuscripts at http://www.hindawi.com
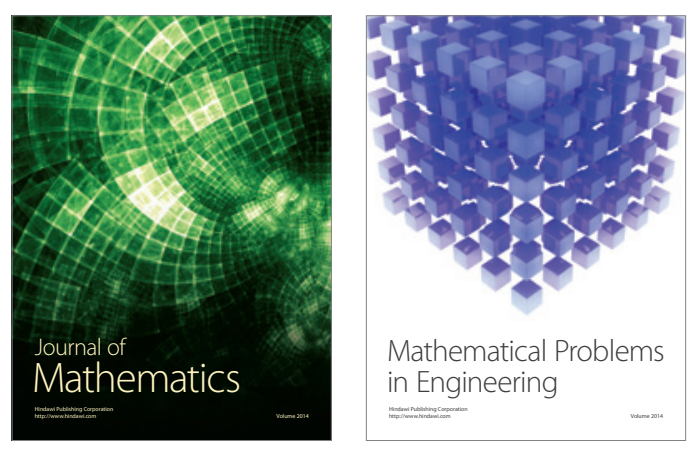

Mathematical Problems in Engineering
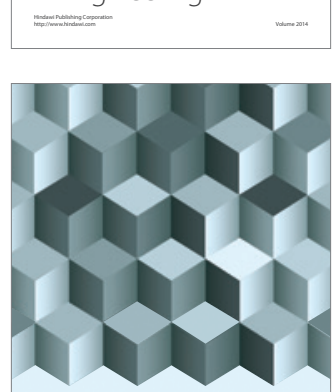

Journal of

Function Spaces
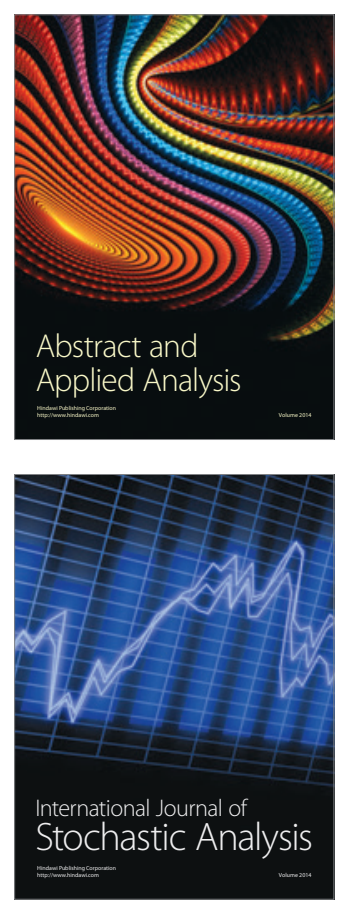

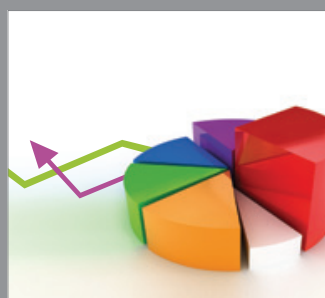

ournal of

Probability and Statistics

Promensencen
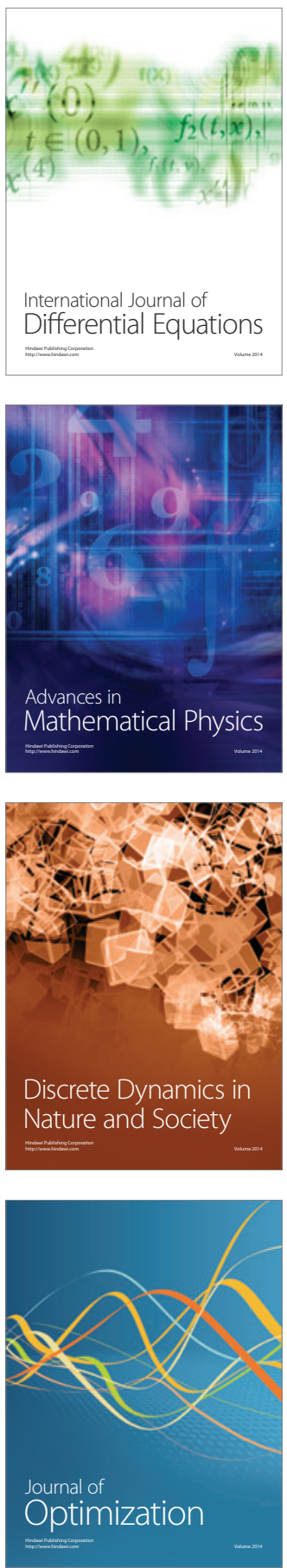